\title{
On convergence of the Kähler-Ricci flow
}

\author{
Ovidiu Munteanu and Gábor SzÉKelyhidi
}

\begin{abstract}
We study the convergence of the Kähler-Ricci flow on a Fano manifold under some stability conditions. More precisely we assume that the first eingenvalue of the $\bar{\partial}$-operator acting on vector fields is uniformly bounded along the flow, and in addition the Mabuchi energy decays at most logarithmically. We then give different situations in which the condition on the Mabuchi energy holds.
\end{abstract}

\section{Introduction}

Let $X$ be a compact Kähler manifold of dimension $n$ with $c_{1}(X)>0$ and let us consider the Ricci flow introduced by Hamilton [9] defined by

$$
\begin{aligned}
\frac{\partial}{\partial t} g_{i \bar{j}} & =-\left(R_{i \bar{j}}-g_{i \bar{j}}\right), \\
g_{i \bar{j}}(0) & \in c_{1}(X) .
\end{aligned}
$$

Cao [3] showed that this flow exists for all time. When $c_{1}(X)=0$ or $c_{1}(X)<$ 0, then Cao also showed, using Yau's estimates [23], that the analogous normalized flow converges to a Kähler-Einstein metric on $X$. When $c_{1}(X)>$ 0 , i.e. the manifold is Fano, then it is still an open problem to determine whether $X$ admits a Kähler-Einstein metric. The central problem in the field is the following conjecture.

Conjecture 1.1 Yau-Tian-Donaldson Conjecture. A Fano manifold $X$ admits a Kähler-Einstein metric if and only if it is K-polystable.

For more details see [7, 20] and also [13] for a survey and many more references. If we try to use the Kähler-Ricci flow to find a Kähler-Einstein metric then in light of this conjecture the key problem is to relate $K$-polystability of $X$ to convergence of the flow. This still seems out of reach at present, but many partial results have been obtained. One possibility is to assume that the Riemann curvature tensor remains uniformly bounded along the flow, for results in this direction see $[4,11,14,17,21]$. 
In this paper, we study a different kind of assumption, which was introduced by Phong-Song-Sturm-Weinkove in [12]. The main assumption is that along the flow $g(t)$ there is a uniform lower bound $\lambda_{t} \geqslant \lambda>0$ on the lowest positive eigenvalue $\lambda_{t}$ of the $\bar{\partial}$-operator acting on vector fields. The main result in [12] is that if in addition we assume that the Mabuchi energy is bounded from below, then the flow converges to a Kähler-Einstein metric. Our main result is a weakening of the hypothesis on the Mabuchi energy.

Theorem 1.1. Suppose that along the Kähler-Ricci flow we have a uniform lower bound $\lambda_{t} \geqslant \lambda>0$ on the first eingenvalue, and in addition the Mabuchi energy satisfies

$$
\mathcal{M}(g(t))>-C \log (1+t)-D
$$

for some constants $C, D>0$. Then the metrics $g(t)$ converge exponentially fast in $C^{\infty}$ to a Kähler-Einstein metric.

In fact the proof of the theorem shows that instead of (1.1) it is enough to assume

$$
\inf _{t>0} Y(t)=0
$$

where

$$
Y(t)=-\frac{d}{d t} \mathcal{M}(g(t))
$$

Since the Mabuchi energy is monotonically decreasing under the KählerRicci flow, the assumption of the theorem clearly implies this weaker statement. We will prove this theorem in Section 3. A first step is to show that the Futaki invariant of the manifold vanishes, which we prove in Lemma 3.1. The main novelty is the estimate in Lemma 3.2.

The advantage of replacing the lower bound on $\mathcal{M}$ by these weaker statements is that there are some other natural conditions under which they can be shown to hold. We give two such conditions.

In Section 4, we show directly that on a $K$-semistable toric variety the condition (1.1) of Theorem 1.1 holds.

Theorem 1.2. Suppose that the Fano toric variety $X$ is K-semistable. If $g(t)$ are torus invariant metrics satisfying the Kähler-Ricci flow then

$$
\mathcal{M}(g(t))>-C \log (1+t)-D
$$

for some constants $C, D>0$. 
While it is already known that $K$-semistable toric varieties admit KählerEinstein metrics (see [22]) and hence the Mabuchi energy is bounded below, this direct argument could be of independent interest.

In Section 5, we show that if we define

$$
R(X):=\sup \left\{t \mid \text { there exists } \omega \in c_{1}(X) \text { such that } \operatorname{Ric}(\omega)>t \omega\right\},
$$

then the condition $R(X)=1$ implies that (1.2) holds.

Theorem 1.3. Suppose that $X$ is a Fano manifold which satisfies $R(X)=$ 1. Then along the Kähler-Ricci flow $g(t)$ on $X$, we have

$$
\inf _{t>0} Y(g(t))=0 \text {. }
$$

As a corollary we note

Corollary 1.1. If $X$ is a Fano manifold with $R(X)=1$ then $X$ is K-semistable.

This is a strengthening of a result in [16], where the second author conjectured that the condition $R(X)=1$ is equivalent to $K$-semistability.

Finally we note that if $X$ is a degree $n+1$ hypersurface in $\mathbf{P}^{n+1}$ and $n+1 \geqslant 3$, then the $\alpha$-invariant of $X$, defined by Tian [19], satisfies (see [5])

$$
\alpha(X) \geqslant \frac{n}{n+1} .
$$

This implies that $R(X)=1$, since given any Kähler metric $\omega_{0}$ and $0<t<1$, we can find $\omega$ that satisfies

$$
\operatorname{Ric}(\omega)=t \omega+(1-t) \omega_{0}
$$

so in particular $\operatorname{Ric}(\omega)>t \omega$ (see [19]). By the above results $X$ is $K$-semistable and also the condition (1.2) holds. Note that it is not known whether the Mabuchi energy is bounded from below for such hypersurfaces.

\section{Background}

In this section, we recall some basic notation and results that we use. We consider the normalized Kähler-Ricci flow

$$
\begin{aligned}
\frac{\partial}{\partial t} g_{i \bar{j}} & =-\left(R_{i \bar{j}}-g_{i \bar{j}}\right)=\partial_{i} \partial_{\bar{j}} u, \\
g_{i \bar{j}}(0) & \in c_{1}(X) .
\end{aligned}
$$


Here $R_{i \bar{j}}$ is the Ricci curvature and $u(t)$ is the Ricci potential of the metric $g(t)$, which we normalize so that

$$
\frac{1}{V} \int_{X} e^{-u} \omega^{n}=1 .
$$

Here

$$
V=\int_{X} \omega^{n} .
$$

A fundamental result of Perelman [10] (see [15] for a detailed exposition) is that there exists a constant $C_{0}$ depending only on $g(0)$ such that

$$
\|u\|_{C^{0}}+\|\nabla u\|_{C^{0}}+\|\Delta u\|_{C^{0}} \leqslant C_{0}
$$

along the flow. We also need to recall the following lemma from [12].

Lemma 2.1. We have the following two results along the Kähler-Ricci flow.

- There exist $\delta, K>0$ depending on the dimension $n$ with the following property. For any $\epsilon$ with $0<\epsilon \leqslant \delta$ and any $t_{0} \geqslant 0$, if

$$
\left\|u\left(t_{0}\right)\right\|_{C^{0}} \leqslant \epsilon
$$

then

$$
\left\|\nabla u\left(t_{0}+2\right)\right\|_{C^{0}}+\left\|R\left(t_{0}+2\right)-n\right\|_{C^{0}} \leqslant K \epsilon .
$$

- There exists $C>0$ depending on $g(0)$ such that

$$
\|u\|_{C^{0}}^{n+1} \leqslant C\|\nabla u\|_{L^{2}}\|\nabla u\|_{C^{0}}^{n} .
$$

Proof. The first part is Lemma 1 from [12]. The second part also follows directly from Lemma 3 in [12]. There it is shown that

$$
\|u-b\|_{C^{0}}^{n+1} \leqslant C\|\nabla u\|_{L^{2}}\|\nabla u\|_{C^{0}}^{n}
$$

where $b$ is the average

$$
b=\frac{1}{V} \int_{X} u \mathrm{e}^{-u} \omega^{n},
$$

and also $|b| \leqslant\|u-b\|_{C^{0}}$. But then

$$
\|u\|_{C^{0}} \leqslant\|u-b\|_{C^{0}}+|b| \leqslant 2\|u-b\|_{C^{0}},
$$

so our statement follows. 
We define the Mabuchi functional $\mathcal{M}$ so that $\mathcal{M}(g(0))=0$ and

$$
\frac{d}{d t} \mathcal{M}(g(t))=-\int_{X} u(R-n) \omega^{n}=-\int_{X}|\nabla u|^{2} \omega^{n}
$$

where $R$ is the scalar curvature of the metric $g(t)$. In particular $\mathcal{M}$ is monotonically decreasing under the flow. We define

$$
Y(t)=\int_{X}|\nabla u|^{2} \omega^{n} .
$$

The key to proving convergence of the Kähler-Ricci flow is showing exponential decay of $Y(t)$ (see [12]). For this the basic inequality proved in [14] is

$$
\begin{aligned}
\frac{d}{d t} Y(t) \leqslant & -2 \lambda_{t} Y(t)-2 \lambda_{t} \operatorname{Fut}\left(\pi_{t}\left(\nabla^{j} u\right)\right)-\int_{X}|\nabla u|^{2}(R-n) \omega^{n} \\
& -\int_{X} \nabla^{j} u \nabla^{\bar{k}} u\left(R_{j \bar{k}}-g_{j \bar{k}}\right) \omega^{n}
\end{aligned}
$$

where Fut $\left(\pi_{t}\left(\nabla^{j} u\right)\right)$ is the Futaki invariant, applied to the orthogonal projection $\pi_{t}\left(\nabla^{j} u\right)$ of the vector field $\nabla^{j} u$ on the space of holomorphic vector fields.

\section{The main argument}

Our goal is to prove the following result.

Theorem 3.1. Assume that the lowest positive eigenvalue of the Laplacian $-g^{j \bar{k}} \nabla_{j} \nabla_{\bar{k}}$ acting on $T^{1,0}$ vector fields has a positive lower bound and that the Mabuchi energy along the Kähler-Ricci flow satisfies

$$
\mathcal{M}(g(t))>-C \log (1+t)-D
$$

for some constants $C, D>0$. Then the metrics $g_{i \bar{j}}$ converge exponentially fast in $C^{\infty}$ to a Kähler-Einstein metric.

Before proving the theorem we need two Lemmas.

Lemma 3.1. If $\inf _{t \geqslant 0} Y(t)=0$, then the Futaki invariant of $X$ vanishes. 
Proof. Using the second part of Lemma 2.1, we have

$$
\|u\|_{C^{0}}^{n+1}(t) \leqslant C\|\nabla u\|_{L^{2}}(t)\|\nabla u\|_{C^{0}}^{n}(t) \leqslant C_{1} Y(t)^{1 / 2}
$$

where we have also used Perelman's estimate (2.1). It follows that $\inf _{t \geqslant 0}\|u\|_{C^{0}}=0$. Then the first part of Lemma 2.1 implies that

$$
\inf _{t \geqslant 0}\|R-n\|_{C^{0}}=0
$$

Thus the manifold has to be $K$-semistable by Donaldson's lower bound [8]. In particular, the Futaki invariant of $X$ vanishes.

Lemma 3.2. On any Kähler manifold $X$ such that

$$
R_{i \bar{j}}-g_{i \bar{j}}=-u_{i \bar{j}}
$$

we have the following estimate

$$
\left|\int_{X} u_{j \bar{k}} \nabla^{j} u \nabla^{\bar{k}} u\right| \leqslant 5\left(\|\nabla u\|_{C^{0}}^{2}+\|\Delta u\|_{C^{0}}\right) \int_{X}|\nabla u|^{2} .
$$

Proof. We have

$$
\begin{aligned}
\left|\int_{X} u_{j \bar{k}} \nabla^{j} u \nabla^{\bar{k}} u\right| & \leqslant \int_{X}\left|u_{j \bar{k}}\right||\nabla u|^{2} \\
& \leqslant\left(\int_{X}\left|u_{j \bar{k}}\right|^{2}|\nabla u|^{2}\right)^{1 / 2}\left(\int_{X}|\nabla u|^{2}\right)^{1 / 2} .
\end{aligned}
$$

We now denote

$$
I=\int_{X}\left|u_{j \bar{k}}\right|^{2}|\nabla u|^{2}
$$

Integration by parts yields:

$$
\begin{aligned}
I & =\int_{X} u_{j \bar{k}} u_{k \bar{j}}|\nabla u|^{2} \\
& =-\int_{X} u_{j \bar{k} \bar{j}} u_{k}|\nabla u|^{2}-\int_{X} u_{j \bar{k}} u_{k}\left(|\nabla u|^{2}\right)_{\bar{j}} \\
& \leqslant\left.\left|\int_{X} u_{j \bar{k} \bar{j}} u_{k}\right| \nabla u\right|^{2}|+| \int_{X} u_{j \bar{k}} u_{k}\left(|\nabla u|^{2}\right)_{\bar{j}} \mid .
\end{aligned}
$$


The first term above is, using the Ricci identities,

$$
\begin{aligned}
& \left.\left|\int_{X} u_{j \bar{k} \bar{j}} u_{k}\right| \nabla u\right|^{2} \mid \\
& \quad=\left.\left|\int_{X}(\Delta u)_{\bar{k}} u_{k}\right| \nabla u\right|^{2} \mid \\
& \quad \leqslant \int_{X}(\Delta u)^{2}|\nabla u|^{2}+\left|\int_{X}(\Delta u) u_{k}\left(|\nabla u|^{2}\right)_{\bar{k}}\right| \\
& \quad \leqslant\|\Delta u\|_{C^{0}}^{2} \int_{X}|\nabla u|^{2}+\int_{X}|\Delta u||\nabla u|\left|\nabla\left(|\nabla u|^{2}\right)\right| \\
& \quad \leqslant\|\Delta u\|_{C^{0}}^{2} \int_{X}|\nabla u|^{2}+\frac{1}{2} \int_{X}(\Delta u)^{2}|\nabla u|^{2}+\frac{1}{2} \int_{X}\left|\nabla\left(|\nabla u|^{2}\right)\right|^{2} \\
& \quad \leqslant \frac{3}{2}\|\Delta u\|_{C^{0}}^{2} \int_{X}|\nabla u|^{2}+\frac{1}{2} \int_{X}\left|\nabla\left(|\nabla u|^{2}\right)\right|^{2} \cdot
\end{aligned}
$$

The second term can be estimated as follows:

$$
\begin{aligned}
\left|\int_{X} u_{j \bar{k}} u_{k}\left(|\nabla u|^{2}\right)_{\bar{j}}\right| & \leqslant \int_{X}\left|u_{j \bar{k}}\right||\nabla u|\left|\nabla\left(|\nabla u|^{2}\right)\right| \\
& \leqslant \frac{1}{2} \int_{X}\left|u_{j \bar{k}}\right|^{2}|\nabla u|^{2}+\frac{1}{2} \int_{X}\left|\nabla\left(|\nabla u|^{2}\right)\right|^{2} \\
& =\frac{1}{2} I+\frac{1}{2} \int_{X}\left|\nabla\left(|\nabla u|^{2}\right)\right|^{2} .
\end{aligned}
$$

Using these estimates in (3.2) it follows that

$$
I \leqslant 3\|\Delta u\|_{C^{0}}^{2} \int_{X}|\nabla u|^{2}+2 \int_{X}\left|\nabla\left(|\nabla u|^{2}\right)\right|^{2} .
$$

We now denote

According to the Bochner formula,

$$
\begin{aligned}
J & =\int_{X}\left|\nabla\left(|\nabla u|^{2}\right)\right|^{2} \\
& =-\int_{X}|\nabla u|^{2} \Delta|\nabla u|^{2} .
\end{aligned}
$$

$$
\Delta|\nabla u|^{2}=2\langle\nabla u, \nabla(\Delta u)\rangle+R_{i \bar{j}} u_{\bar{\imath}} u_{j}+\left|u_{i \bar{j}}\right|^{2}+\left|u_{i j}\right|^{2} .
$$

Note that

$$
\begin{aligned}
R_{i \bar{j}} u_{\bar{\imath}} u_{j} & =\left(g_{i \bar{j}}-u_{i \bar{j}}\right) u_{\bar{\imath}} u_{j} \\
& \geqslant-u_{i \bar{j}} u_{\bar{\imath}} u_{j} \\
& \geqslant-\left|u_{i \bar{j}}\right|^{2}-\frac{1}{4}|\nabla u|^{4},
\end{aligned}
$$


where the last inequality follows from

$$
\begin{aligned}
\left|u_{i \bar{j}} u_{\bar{\imath}} u_{j}\right| & \leqslant\left|u_{i \bar{j}}\right||\nabla u|^{2} \\
& \leqslant\left|u_{i \bar{j}}\right|^{2}+\frac{1}{4}|\nabla u|^{4} .
\end{aligned}
$$

This proves that

$$
\Delta|\nabla u|^{2} \geqslant 2\langle\nabla u, \nabla(\Delta u)\rangle-\frac{1}{4}|\nabla u|^{4} .
$$

We use this to estimate $J$ from above:

$$
\begin{aligned}
J & =-\int_{X}|\nabla u|^{2} \Delta|\nabla u|^{2} \\
& \leqslant-2 \int_{X}\langle\nabla u, \nabla(\Delta u)\rangle|\nabla u|^{2}+\frac{1}{4} \int_{X}|\nabla u|^{4}|\nabla u|^{2} .
\end{aligned}
$$

Let us study the first term in (3.4). We have

$$
\begin{aligned}
- & 2 \int_{X}\langle\nabla u, \nabla(\Delta u)\rangle|\nabla u|^{2} \\
& =2 \int_{X}(\Delta u)^{2}|\nabla u|^{2}+2 \int_{X}(\Delta u)\left\langle\nabla u, \nabla\left(|\nabla u|^{2}\right)\right\rangle \\
& \leqslant 2\|\Delta u\|_{C^{0}}^{2} \int_{X}|\nabla u|^{2}+2 \int_{X}|\Delta u||\nabla u|\left|\nabla\left(|\nabla u|^{2}\right)\right| \\
& \leqslant 2\|\Delta u\|_{C^{0}}^{2} \int_{X}|\nabla u|^{2}+2 \int_{X}|\Delta u|^{2}|\nabla u|^{2}+\frac{1}{2} \int_{X}\left|\nabla\left(|\nabla u|^{2}\right)\right|^{2} \\
& \leqslant 4\|\Delta u\|_{C^{0}}^{2} \int_{X}|\nabla u|^{2}+\frac{1}{2} J .
\end{aligned}
$$

Plugging this into (3.4) we get

$$
J \leqslant 8\|\Delta u\|_{C^{0}}^{2} \int_{X}|\nabla u|^{2}+\frac{1}{2}\|\nabla u\|_{C^{0}}^{4} \int_{X}|\nabla u|^{2} .
$$

We plug this into (3.3) and obtain

$$
I \leqslant\left(19\|\Delta u\|_{C^{0}}^{2}+\|\nabla u\|_{C^{0}}^{4}\right) \int_{X}|\nabla u|^{2} .
$$

Using this in (3.1) we obtain the result.

We can now prove the theorem. 
Proof of Theorem 3.1. Note first of all that our hypothesis implies that

$$
\inf _{t \geqslant 0} Y(t)=0 .
$$

For if $Y(t)>\epsilon>0$ for all $t$ then by Equation (2.2)

$$
\mathcal{M}(g(t))<-\epsilon t,
$$

which contradicts our assumption.

Our goal is to prove that $Y(t)$ has exponential decay, since then the exponential convergence of the Kähler-Ricci flow follows like in [12]. In the inequality (2.3), we use our hypothesis that $\lambda_{t} \geqslant \lambda>0$ and that the Futaki invariant vanishes by Lemma 3.1. We obtain

$$
\frac{d}{d t} Y(t) \leqslant-2 \lambda Y(t)-\int_{X}|\nabla u|^{2}(R-n) \omega^{n}-\int_{X} \nabla^{j} u \nabla^{\bar{k}} u\left(R_{j \bar{k}}-g_{j \bar{k}}\right) \omega^{n} .
$$

Now using Lemma 3.2 and the fact that $R-n=-\Delta u$ we get

$$
\frac{d}{d t} Y(t) \leqslant-2 \lambda Y(t)+6\left(\|\Delta u\|_{C^{0}}(t)+\|\nabla u\|_{C^{0}}^{2}(t)\right) Y(t) .
$$

We remark that formula (3.7) can be used as a substitute for the differentialdifference inequality (5.5) in [12].

We are now ready to finish the proof of the theorem. Fix $\varepsilon_{0}>0$ small to be determined later. There must exist a point $t_{0}>0$ such that

$$
Y\left(t_{0}\right) \leqslant \varepsilon_{0}
$$

because of (3.5). It follows from (3.7) and Perelman's estimates on $\nabla u$ and $\Delta u$ that

$$
\frac{d}{d t} Y(t) \leqslant C Y(t)
$$

for some constant $C>0$. Hence, $Y$ has at most exponential growth and it follows that

$$
Y\left(t_{0}+2\right) \leqslant Y\left(t_{0}\right) \mathrm{e}^{2 C} \leqslant \varepsilon_{0} \mathrm{e}^{2 C} .
$$

Consequently, if we set

$$
\varepsilon_{1}=2 \varepsilon_{0} \mathrm{e}^{2 C},
$$

then for $t \in\left[t_{0}, t_{0}+2\right]$ we have

$$
Y(t) \leqslant \frac{1}{2} \varepsilon_{1} .
$$


Assume that there exists a time $t_{0}<t<\infty$ for which $Y(t)>\varepsilon_{1}$. Then let

$$
t_{1}=\inf \left\{t \mid t>t_{0}, \text { and } Y(t)=\varepsilon_{1}\right\}
$$

be the first time after $t_{0}$ such that $Y\left(t_{1}\right)=\varepsilon_{1}$. By (3.8) we have $t_{1}>t_{0}+2$ and so by the definition of $t_{1}$ we have $Y\left(t_{1}-2\right)<\varepsilon_{1}$. By the second part of Lemma 2.1

$$
\|u\|_{C^{0}}\left(t_{1}-2\right) \leqslant C \varepsilon_{1}^{1 / 2(n+1)} .
$$

Moreover if $\varepsilon_{1}$ is sufficiently small, the first part of Lemma 2.1 gives that

$$
\|\Delta u\|_{C^{0}}\left(t_{1}\right)+\|\nabla u\|_{C^{0}}^{2}\left(t_{1}\right) \leqslant C^{\prime} \varepsilon_{1}^{1 / 2(n+1)} .
$$

Denote

$$
\varepsilon=6 C^{\prime} \varepsilon_{1}^{1 / 2(n+1)},
$$

then (3.7) implies that

$$
\left(\frac{d}{d t} Y\right)\left(t_{1}\right) \leqslant-2 \lambda Y\left(t_{1}\right)+\varepsilon Y\left(t_{1}\right) .
$$

Choose $\varepsilon_{0}$ small enough so that $\varepsilon<\lambda$. Then we have

$$
\left(\frac{d}{d t} Y\right)\left(t_{1}\right) \leqslant-\lambda Y\left(t_{1}\right)=-\lambda \varepsilon_{1}<0
$$

which shows that $Y$ is decreasing in a neighborhood of $t_{1}$, and contradicts the choice of $t_{1}$. The contradiction came from our assumption that there exists finite $t$ such that $Y(t)>\varepsilon_{1}$. Therefore, for all $t>t_{0}$ we must have

$$
Y(t) \leqslant \varepsilon_{1}
$$

Then as above for any $t \geqslant t_{0}+2$ we have

$$
\left(\frac{d}{d t} Y\right)(t) \leqslant-\lambda Y(t)
$$

This shows that $Y(t)$ is exponentially decreasing and therefore by the argument in [12] we get the exponential convergence of the Kähler-Ricci flow to a Kähler-Einstein metric. 


\section{The case of toric varieties}

In this section, we prove the following.

Theorem 4.1. Suppose that the Fano toric variety $X$ is K-semistable. If $g(t)$ are torus invariant metrics satisfying the Kähler-Ricci flow then

$$
\mathcal{M}(g(t))>-C \log (1+t)-D
$$

for some constants $C, D>0$.

In fact, if $X$ is $K$-semistable, then its Futaki invariant must vanish, so by Wang and Zhu [22] $X$ admits a Kähler-Einstein metric. Then BandoMabuchi [2] implies that the Mabuchi energy is bounded below. So a stronger result follows easily from known results, but the interest lies in our more direct proof which uses $K$-semistability explicitly. The proof follows the argument in [18] for the Calabi flow where also more details can be found.

Proof. Suppose that the torus invariant metrics $g(t)$ satisfy the KählerRicci flow. On the dense complex torus $\left(\mathbf{C}^{*}\right)^{n} \subset X$, we have $g(t)=\mathrm{i} \partial \bar{\partial} \phi(t)$ for some torus invariant functions $\phi(t)$. We can therefore think of them as functions on Euclidean space:

$$
\phi(t): \mathbf{R}^{n} \rightarrow \mathbf{R} .
$$

These Kähler potentials $\phi(t)$ satisfy

$$
\frac{\partial}{\partial t} \phi(t)=u(t)=\log \operatorname{det}\left(\phi_{i j}\right)+\phi
$$

where $u$ is the Ricci potential as before. For each $t$ the symplectic potential $f(t)$ is the Legendre transform of $\phi(t)$. Then $f(t)$ is a convex function on a polytope $P$, satisfying the Guillemin boundary conditions (for more details see $[7])$. We have

$$
\frac{\partial}{\partial t} f=-L(f)=\log \operatorname{det}\left(f_{i j}\right)+f-x \cdot \nabla f,
$$

where $x$ is the Euclidean coordinate on the polytope $P$ and the function $L(f)$ is just the Ricci potential expressed in the $x$ coordinates. Let $g$ be a fixed symplectic potential, and define the functional

$$
\mathcal{F}(f)=-\int_{P} \log \operatorname{det}\left(g^{i k} f_{k j}\right) d \mu+\int_{P} g^{i j} f_{i j} d \mu .
$$


Then

$$
\frac{d}{d t} \mathcal{F}(f)=\int_{P} f^{i j} L(f)_{i j} d \mu-\int_{P} g^{i j} L(f)_{i j} d \mu=\int_{P}\left(f^{i j}-g^{i j}\right) L(f)_{i j} d \mu .
$$

When integrating by parts the boundary terms vanish, so

$$
\frac{d}{d t} \mathcal{F}(f)=\int_{P}\left[\left(f^{i j}\right)_{, i j}-\left(g^{i j}\right)_{, i j}\right] L(f) d \mu=\int_{P}(R(g)-R(f)) L(f) d \mu,
$$

where $R(f), R(g)$ are the scalar curvatures of the metrics determined by $f, g$. By Perelman's estimates $R(f)$ and the Ricci potential $L(f)$ (normalized by adding a constant) are uniformly bounded along the flow, so we obtain

$$
\frac{d}{d t} \mathcal{F}(f(t))<C_{1}
$$

i.e.

$$
\mathcal{F}(f(t))<C_{1} t+C_{2}
$$

for some constants $C_{1}, C_{2}$.

Applying the inequality $\log x<x / 2$ to each eigenvalue, we obtain $\log \operatorname{det}(M) \leqslant \frac{1}{2} \operatorname{Tr}(M)$ for any positive definite matrix $M$. Applying this to the defining formula of $\mathcal{F}$, we get

$$
\mathcal{F}(f) \geqslant \frac{1}{2} \int_{P} g^{i j} f_{i j} d \mu .
$$

The AM-GM inequality implies that

$$
-\log \operatorname{det}\left(g^{i k} f_{k j}\right) \geqslant-n \log \left(g^{i j} f_{i j}\right)
$$

so using the convexity of - log we get

$$
\begin{aligned}
-\int_{P} \log \operatorname{det}\left(g^{i k} f_{k j}\right) d \mu & \geqslant-n \int_{P} \log \left(g^{i j} f_{i j}\right) d \mu \\
& \geqslant-C_{3} \log \int_{P} g^{i j} f_{i j} d \mu-C_{4} \\
& \geqslant-C_{5} \log (1+t)-C_{6}
\end{aligned}
$$

where in the last line we have used (4.1) and (4.2). 
In terms of symplectic potentials the Mabuchi energy is given by

$$
\mathcal{M}(f)=-\int_{P} \log \operatorname{det}\left(f_{i j}\right) d \mu+\int_{\partial P} f d \sigma-n \int_{P} f d \mu,
$$

moreover if the manifold is $K$-semistable then

$$
\int_{\partial P} f d \sigma-n \int_{P} f d \mu \geqslant 0
$$

for all convex functions $f$ (see Donaldson [7]). Therefore, we have

$$
\mathcal{M}(f) \geqslant-\int_{P} \log \operatorname{det}\left(g^{i k} f_{k j}\right) d \mu-\int_{P} \log \operatorname{det}\left(g_{i j}\right) d \mu \geqslant-C \log (1+t)-D
$$

using (4.3). This completes the proof.

\section{The case when $R(X)=1$.}

For a Fano manifold $X$ we define

$$
R(X)=\sup \left\{t \mid \text { there exists a metric } \omega \in c_{1}(X) \text { such that } \operatorname{Ric}(\omega)>t \omega\right\} .
$$

We show the following.

Theorem 5.1. Suppose that $X$ is a Fano manifold which satisfies $R(X)=1$. Then along the Kähler-Ricci flow $g(t)$ on $X$, we have

$$
\inf _{t>0} Y(g(t))=0 \text {. }
$$

Proof. We argue by contradiction. Suppose that

$$
\inf _{t>0} Y(g(t))=\epsilon>0 \text {. }
$$

Since $\frac{d}{d t} \mathcal{M}(g(t))=-Y(g(t))$, we then have

$$
\mathcal{M}(g(t))<-\epsilon t+C
$$

for some constant $C$. Fix a base metric $\omega_{0} \in c_{1}(X)$, and define the $\mathcal{J}$ functional by $\mathcal{J}\left(\omega_{0}\right)=0$ and

$$
\frac{d}{d s} \mathcal{J}\left(\omega_{s}\right)=\int_{X} \dot{\phi}_{s}\left(\Lambda_{\omega_{s}} \omega_{0}-n\right) \omega_{s}^{n}
$$


where $\omega_{s}=\omega_{0}+\mathrm{i} \partial \bar{\partial} \phi_{s}$ is a path of metrics, and $\Lambda_{\omega_{s}} \omega_{0}$ is the trace of the metric $\omega_{0}$ with respect to $\omega_{s}$. Using the path $\omega_{s}=\omega_{0}+\operatorname{si\partial } \bar{\partial} \phi$ we get

$$
\begin{aligned}
\mathcal{J} & \left(\omega_{0}+\mathrm{i} \partial \bar{\partial} \phi\right) \\
& =\int_{0}^{1} \int_{X} \phi\left(\Lambda_{\omega_{s}} \omega_{0}-n\right) \omega_{s}^{n} d s \\
& =n \int_{0}^{1} \int_{X} \phi\left(\omega_{0}-\omega_{s}\right) \wedge \omega_{s}^{n-1} d s \\
& =n \int_{0}^{1} \int_{X} \phi(-s \mathrm{i} \partial \bar{\partial} \phi) \wedge\left(s \omega_{1}+(1-s) \omega_{0}\right)^{n-1} d s \\
& =n \int_{0}^{1} \int_{X} \phi(-\mathrm{i} \partial \bar{\partial} \phi) \wedge \sum_{k=0}^{n-1}\left(\begin{array}{c}
n-1 \\
k
\end{array}\right) s^{k+1}(1-s)^{n-1-k} \omega_{1}^{k} \wedge \omega_{0}^{n-1-k} d s \\
& =n \int_{X} \phi(-\mathrm{i} \partial \bar{\partial} \phi) \wedge \sum_{k=0}^{n-1}\left(\begin{array}{c}
n-1 \\
k
\end{array}\right) \frac{1}{n+1}\left(\begin{array}{c}
n+1 \\
k
\end{array}\right)^{-1} \omega_{1}^{k} \wedge \omega_{0}^{n-1-k} \\
& =\int_{X} \phi(-\mathrm{i} \partial \bar{\partial} \phi) \wedge \sum_{k=0}^{n-1} \frac{n}{n+1} \cdot \frac{k+1}{n} \cdot \omega_{1}^{k} \wedge \omega_{0}^{n-1-k} \\
& \leqslant \frac{n}{n+1} \int_{X} \phi\left(\omega_{0}-\omega_{1}\right) \wedge \sum_{k=0}^{n-1} \omega_{1}^{k} \wedge \omega_{0}^{n-1-k} \\
& =\frac{n}{n+1} \int_{X} \phi\left(\omega_{0}^{n}-\omega_{1}^{n}\right) .
\end{aligned}
$$

This is the well-known inequality $I-J \leqslant \frac{n}{n+1} I$ in the literature in terms of Aubin's $I, J$ functionals (see [1]).

The point is that along the Kähler-Ricci flow $g(t)$ we have $|\dot{\phi}|<C_{1}$ for some constant $C_{1}$ by Perelman's estimates, so it follows that

$$
|\phi(t)|<C_{1} t+C_{2}
$$

It follows that

$$
\mathcal{J}(g(t))<C_{1} t+C_{2}
$$

for some different constants $C_{1}, C_{2}$. But then using (5.1)

$$
\mathcal{M}(g(t))+\frac{\epsilon}{2 C_{1}} \mathcal{J}(g(t))<-\frac{\epsilon}{2} t+C^{\prime}
$$


and in particular the functional $\mathcal{M}+\frac{\epsilon}{2 C_{1}} \mathcal{J}$ is not bounded from below on $c_{1}(X)$. It follows then using the work of Chen-Tian [6] (see [16]) that

$$
R(X) \leqslant 1-\frac{\epsilon}{2 C_{1}}
$$

which is a contradiction.

Finally, we note

Corollary 5.1. If $X$ is a Fano manifold with $R(X)=1$ then $X$ is K-semistable.

Proof. This follows from the previous theorem and the proof of Lemma 3.1.

\section{Acknowledgment}

We would like to thank D. H. Phong for his encouragement and interest in this work.

\section{References}

[1] T. Aubin, Réduction de cas positif de l'équation de Monge-Ampère sur les variétés kählériennes compactes à la démonstration d'une inégalité, J. Funct. Anal. 57(2) (1984), 143-153.

[2] S. Bando and T. Mabuchi, Uniqueness of Einstein Kähler metrics modulo connected group actions, in Algebraic geometry, Sendai (1985), vol. 10 of Adv. Stud. Pure Math., pp. 11-40.

[3] H.D. Cao, Deformation of Kähler metrics to Kähler-Einstein metrics on compact Kähler manifolds, Invent. Math. 81(2) (1985), 359-372.

[4] H.-D. Cao and M. Zhu, A note on compact Kähler-Ricci flow with positive bisectional curvature, Math. Res. Lett. 16(6) (2009), 935-939.

[5] I. Cheltsov and J. Park, Log canonical thresholds and generalized Eckardt points, Sb. Math. 193(5-6) (2002), 779-789.

[6] X.X. Chen and G. Tian, Geometry of Kähler metrics and foliations by holomorphic discs Publ. Math. Inst. Hautes Études Sci. 107 (2008), $1-107$. 
[7] S.K. Donaldson, Scalar curvature and stability of toric varieties, J. Differential Geom. 62 (2002), 289-349.

[8] S.K. Donaldson, Lower bounds on the Calabi functional, J. Differential Geom. 70(3) (2005), 453-472.

[9] R. Hamilton, Three-manifolds with positive Ricci curvature, J. Differential Geom. 17(2) (1982), 255-306.

[10] G. Perelman, The entropy formula for the Ricci flow and its geometric applications, arXiv:math.DG/0211159.

[11] D.H. Phong, J. Song, J. Sturm and B. Weinkove, The Kähler-Ricci flow with positive bisectional curvature, Invent. Math. 173(3) (2008), $651-665$.

[12] D.H. Phong, J. Song, J. Sturm and B. Weinkove, The Kähler-Ricci flow and the $\bar{\partial}$ operator on vector fields, J. Differential Geom. 81(3) (2009), $631-647$.

[13] D.H. Phong and J. Sturm, Lectures on stability and constant scalar curvature, in Current Developments in Mathematics (2007), International Press, arXiv:0801.4179.

[14] D.H. Phong and J. Sturm, On stability and the convergence of the Kähler-Ricci flow, J. Differential Geom. 72(1) (2006), 149-168.

[15] N. Sesum and G. Tian, Bounding scalar curvature and diameter along the Kähler-Ricci flow (after Perelman), J. Inst. Math. Jussieu 7(3) (2008), 575-587.

[16] G. Székelyhidi, Greatest lower bounds on the Ricci curvature of Fano manifolds, Compos. Math. 147 (2011), 319-331, arXiv:0903.5504.

[17] G. Székelyhidi, The Kähler-Ricci flow and K-polystability, Amer. J. Math. 132 (2010), 1077-1090, arXiv:0803.1613.

[18] G. Székelyhidi, Optimal test-configurations for toric varieties, J. Differential Geom. 80(3) (2008), 501-523.

[19] G. Tian, On Kähler-Einstein metrics on certain Kähler manifolds with $c_{1}(M)>0$, Invent. Math. 89 (1987), 225-246.

[20] G. Tian, Kähler-Einstein metrics with positive scalar curvature, Invent. Math. 137 (1997), 1-37. 
[21] V. Tosatti, Kähler-Ricci flow on stable Fano manifolds, J. Reine Angew. Math. 640 (2010), 67-84, arXiv:0810.1895.

[22] X.-J. Wang and X. Zhu, Kähler-Ricci solitons on toric manifolds with positive first Chern class, Adv. Math. 188 (2004), 87-103.

[23] S.-T. Yau, On the Ricci curvature of a compact Kähler manifold and the complex Monge-Ampère equation I, Comm. Pure Appl. Math. 31 (1978), 339-411.

Department of Mathematics

COLUMBia University

NEW YORK, NY 10027

USA

E-mail address: omuntean@math.columbia.edu

Department of Mathematics

University of Notre Dame

Notre Dame, IN 46556

USA

E-mail address: gszekely@nd.edu

Received February 5, 2010 
